\title{
Unicompartmental knee arthroplasty, is it superior to high tibial osteotomy in treating unicompartmental osteoarthritis? A meta-analysis and systemic review
}

\author{
Marcel Budhi Santoso and Lidong Wu
}

\begin{abstract}
Background: Debate remains whether high tibial osteotomy (HTO) or unicompartmental knee arthroplasty (UKA) is more beneficial for the treatment of unicompartmental knee osteoarthritis. The purpose of this study was to compare the functional results, knee scores, activity levels, and complications between the two procedures.

Methods: We performed a systematic review of published literature from August 1982 through January 2017. Fifteen papers reporting three prospective randomized trials were subjected to a meta-analysis.

Results: No significant difference between the two groups was noted with respect to free walking (velocity), knee score, deterioration of the contralateral or patellofemoral knee, or revision rate and total knee arthroplasty. However, UKA produced better outcomes compared to HTO in terms of the functional results, pain assessment, and complications, although patients who underwent HTO tended to have slightly better range of motion.

Conclusions: Valgus HTO provides better physical activity for younger patients whereas UKA is more suitable for older patients due to shorter rehabilitation time and faster functional recovery. Although UKA patients tended to have improved overall long-term outcomes, which may be due to accurate indications and patient selection, both treatment options yielded pleasing results. Therefore, we are unable to conclude that either method is superior.
\end{abstract}

Keywords: High tibial osteotomy (HTO), Unicompartmental knee arthroplasty (UKA), Osteoarthritis, Meta-analysis

\section{Background}

The management of degenerative osteoarthritis (OA) aims to provide symptomatic relief and to promote knee function, which may be done conservatively or by means of high tibial osteotomy (HTO) or knee replacement arthroplasty.

HTO is a globally recognized treatment option for medial compartment OA of the knee, particularly for patients who are young and active. This procedure was first conducted in 1958 [1] to correct a varus deformity by lateral mechanical axis relocation $[2,3]$. Patients receiving HTO can benefit from natural joint preservation, with physical loading being almost completely unaffected.

\footnotetext{
* Correspondence: wulidong@zju.edu.cn

Department of Orthopedic Surgery, The Second Affiliated Hospital, School of Medicine, Zhejiang University, 88th Jiefang Road, Hangzhou 310009,

Zhejiang Province, People's Republic of China
}

Unicompartmental knee arthroplasty (UKA) was first introduced in the 1970s [4] as an alternative to total knee arthroplasty (TKA) or HTO for singlecompartment OA. UKA is a joint resurfacing procedure in which the affected degenerative compartment is treated with an implant prosthesis, while the nonaffected compartment is preserved. UKA allows knee bone stock preservation and offers patients a less invasive procedure with a faster recovery time [5].

Studies that compare the outcomes of HTO and UKA and their effects are lacking; thus, the relative merits of the two procedures are still under debate. The aim of this study was to evaluate both procedures for the treatment of unicompartmental knee OA using recent reports concerning the indications, functional outcomes, complications, and subsequent revisions to TKA after failed HTO or UKA. 


\section{Methods}

\section{Search strategy}

The present study was conducted using the Preferred Reporting Items for Systematic Reviews and MetaAnalyses (PRISMA) statement. A computerized search of electronic databases (MEDLINE, Embase, and Cochrane) for English-language studies, as well as all related published full studies prior to January 2017, was performed using the following keywords to maximize the search sensitivity and specificity: "high tibial osteotomy (HTO)," "unicompartmental knee arthroplasty (UKA)," "unicompartmental knee osteoarthritis," and "high tibial osteotomy versus unicompartmental knee arthroplasty."

\section{Inclusion and exclusion criteria}

All retrospective studies and prospective randomized studies that satisfied the search strategy were reviewed and were included in the present analysis if they met the following criteria: studies comparing the outcomes of HTO and UKA that clearly described at least one of the indices investigated in this analysis, articles published in English, and cases with no previous history of knee injury. The title and abstract were examined independently by two reviewers. All disagreements were resolved through discussion until a consensus was reached.

\section{Data collection}

All information regarding participants and clinical outcomes was recorded. Participant data included the number of patients, age, gender, and number of knees treated. The principle outcomes of interest included post-operative functional outcomes, range of motion, velocity, complications, and incidence of revision to TKA. Data were documented independently by two authors after the qualifying studies were selected.

\section{Quality assessment}

The reliability of results depends on the extent to which potential sources of bias have been avoided. To adopt the same method to evaluate all selected studies, two reviewers independently applied the "assessing risk of bias" table to assess the risk of bias in each included study. The following biases were assessed: selection bias, performance bias, attrition bias, detection bias, reporting bias, and other bias. Disagreements were resolved through discussion between the reviewers.

\section{Statistical analysis}

The heterogeneity of this study was determined by documenting the methodological distinctions among several studies by analyzing the data extraction tables. The $I^{2}$ test was used to evaluate statistical heterogeneity; if the $P$ value was less than 0.05 and the $I^{2}$ value was less than $50 \%$, a fixed-effects model was selected. However, in cases where these conditions were not satisfied, a random-effects model was adopted [6].

The odds ratio (OR) and associated 95\% confidence interval $(\mathrm{CI})$ were used to determine the value of dichotomous data. Continuous data were evaluated by means of the standardized mean difference (STD) and the corresponding 95\% CI values using the Mantel-Haenszel method [7].

In all cases, $P$ values $<0.05$ were considered statistically significant. Sensitivity and subgroup analyses were conducted to obtain a solid conclusion and to evaluate the stability of the results. Review Manager (RevMan) version 5.3 for Windows and the Cochrane collaboration were used to interpret the relevant variables and establish the $95 \%$ CI.

\section{Results}

\section{Study characteristic}

A total of 1723 titles and abstracts were identified using the search strategies described above, of which 1481 were full-text publications that were then screened based on the inclusion criteria. Thirty-nine studies compared HTO and UKA; however, 24 studies were excluded (Fig. 1). Ultimately, 15 studies [5, 8-21] were selected and included in our analysis, of which only 3 were prospective randomized studies (Fig. 2).

\section{Population characteristic}

Overall, 1013 patients/1041 knees were treated with HTO and 5438 patients $/ 5497$ knees were treated with UKA. Patients' age ranges were 42.7-71 years and 49.2-80 years, respectively. Only 11 studies [5, 8, 9, 12, 13, 15, 17-21] provided patients' gender: 195 males and 277 females underwent HTO, whereas 182 males and 374 females underwent UKA. The follow-up period ranged from a minimum of 0.5 years to a maximum of 17 years. Eight studies $[5,12,13,15,17,19-21]$ reported the indications for inclusion in the study; these were strictly used for isolated medial knee OA with a varus deformity. The inclusion criteria for the other studies were varied or unclear. Six papers $[5,12,13,15,18,20]$ reported the cohorts using the Ahlbäck OA score, and three papers $[9,17,21]$ used the Kellgreen-Lawrence (K/L) score. All of the included studies described the type of procedure, except one [18] in the HTO group and three $[8,16,18]$ in the UKA group. Details are provided in Tables 1 and 2.

\section{Meta-analysis}

Because the measurement time points varied among studies, nearly all results reported here reflect the pooled data without period stratification. In addition, not all studies presented the essential data, introducing a potential bias to this study. Upon analyzing statistical heterogeneity, seven outcomes showed substantial heterogeneity $\left(I^{2}\right.$ values $\left.>50 \%\right)$ and were therefore interpreted with caution (Table 3 ). 


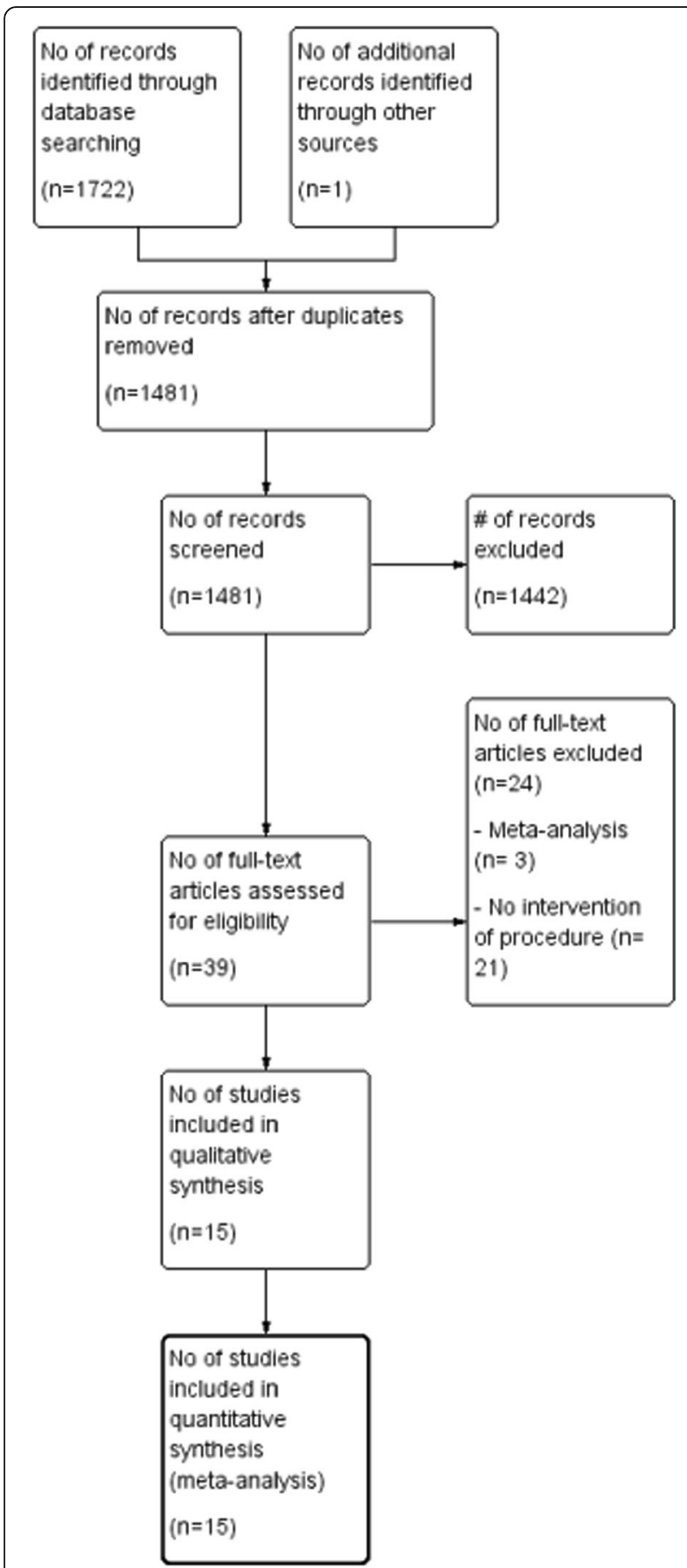

Fig. 1 PRISMA Chart

There was limited evidence of a publication bias, with a broad symmetrical funnel plot assessing the primary outcomes (excellent/good results) (Fig. 3).

\section{Primary outcome}

The analysis of 10 studies $[5,8,9,11-15,18,20]$ yielded a statistically significant difference between HTO and UKA regarding excellent/good results $(p<0.001 ; \mathrm{OR}=0.37$;

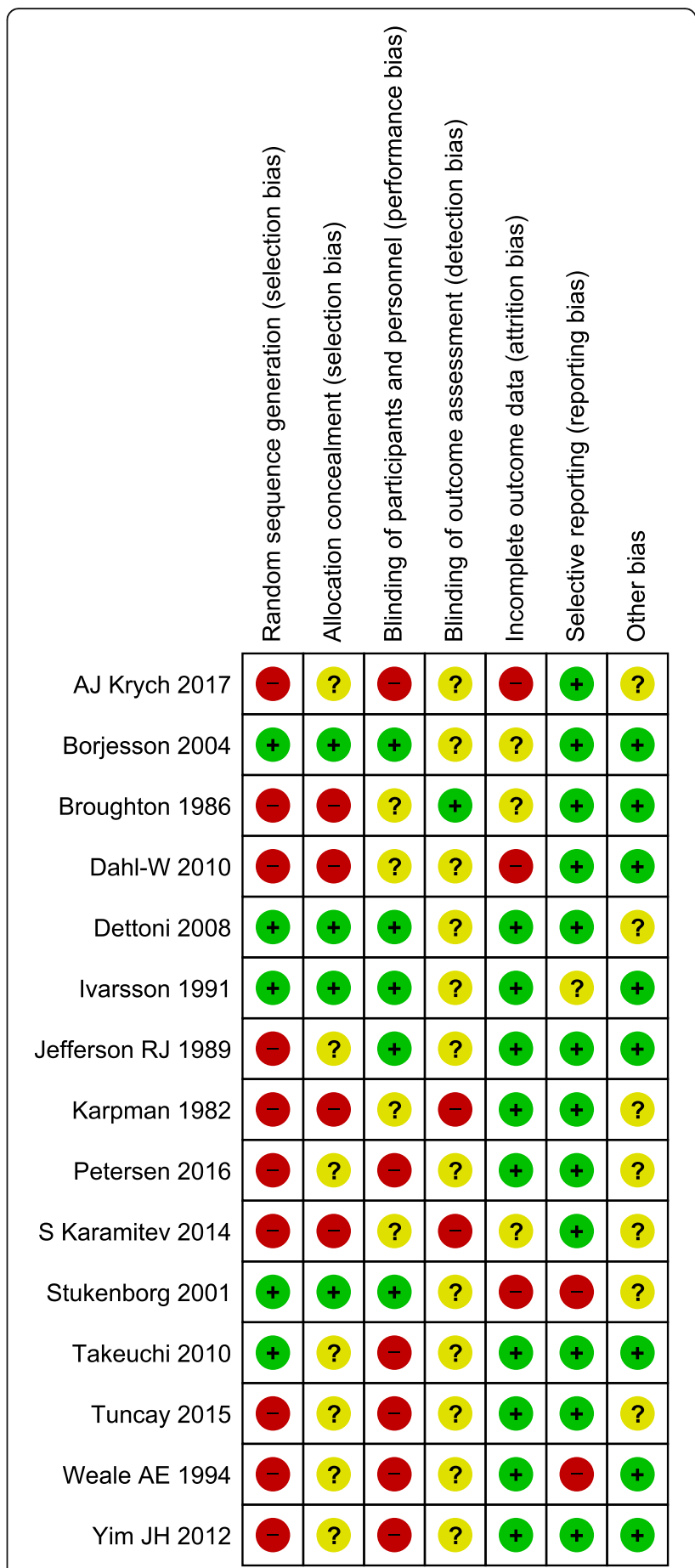

Fig. 2 Risk of bias assessment shown in included studies

95\% CI =0.24, 0.58; Fig. 4). Among them, five studies $[5,12,13,15,20]$ provided clear support for medial OA, specifically in cases of varus deformity; however, the difference was not significant $(p=0.43$; OR $=0.75$; $95 \% \mathrm{CI}=0.37,1.52)$. Moreover, the subgroup analysis for the HTO group included opening $[14,15,20]$ and closing-wedge $[5,8,9,11-13]$ procedures; these 
Table 1 Description of studies included in the meta-analysis

\begin{tabular}{|c|c|c|c|c|c|c|c|c|c|}
\hline Author & Year & Type of study & Type & Pts & Knee & $M / F$ & Age (years) & $\begin{array}{l}\text { HTO type/ } \\
\text { UKA model }\end{array}$ & Follow-up \\
\hline \multirow[t]{2}{*}{ Karpman et al. [8] } & 1982 & Retrospective & HTO & 21 & 23 & $18 / 3$ & 57 & CWHTO & 2 years \\
\hline & & & UKA & 19 & 21 & $15 / 4$ & 62 & NS & 3 years \\
\hline \multirow[t]{2}{*}{ Broughton et al. [9] } & 1986 & Retrospective & HTO & 45 & 49 & $11 / 38$ & 71 & CWHTO & 7.8 years \\
\hline & & & UKA & 34 & 42 & $11 / 31$ & 63 & St Georg & 5.8 years \\
\hline Jefferson RJ et al. & 1989 & Prospective & HTO & 20 & 23 & NS & 57 & CWHTO & NS \\
\hline [10] & & & UKA & 20 & 24 & & 65 & Oxford & \\
\hline \multirow[t]{2}{*}{ Ivarsson et al. [5] } & 1991 & Prospective & HTO & 10 & 10 & $4 / 6$ & 62 & CWHTO & 1 year \\
\hline & & Randomized & UKA & 10 & 10 & $4 / 6$ & 64 & Oxford/PCA & 0.5 years \\
\hline \multirow[t]{2}{*}{ Weale et al. [11] } & 1994 & Retrospective & HTO & 21 & 21 & NS & 74 & CWHTO & $12-17$ years \\
\hline & & & UKA & 15 & 15 & & 80 & St Georg & $12-17$ years \\
\hline Stukenborg et al. & 2001 & Prospective & HTO & 32 & 32 & 19/13 & 67 & CWHTO & 7.5 years \\
\hline [12] & & Randomized & UKA & 28 & 30 & $6 / 22$ & 67 & Aesculap & 7.5 years \\
\hline \multirow[t]{2}{*}{ Borjesson et al.[13] } & 2004 & Prospective & HTO & 18 & 18 & $10 / 8$ & 63 & CWHTO & 5 years \\
\hline & & Randomized & UKA & 22 & 22 & $11 / 11$ & 63 & Brigham & 5 years \\
\hline \multirow[t]{2}{*}{ Dettoni et al. [14] } & 2008 & Prospective & HTO & 54 & & NS & NS & OWHTO & $2-4$ years \\
\hline & & & UKA & 56 & & & & Accuris & $2-4$ years \\
\hline \multirow[t]{2}{*}{ Takeuchi et al. [15] } & 2010 & Retrospective & HTO & 24 & 27 & $6 / 18$ & 67 & OWHTO & 5.1 years \\
\hline & & & UKA & 18 & 30 & $4 / 14$ & 77 & Nakashima & 7 years \\
\hline \multirow[t]{2}{*}{ Dahl-W et al. [16] } & 2010 & Registry & HTO & 450 & & NS & NS & Hemicallotasis & NS \\
\hline & & Review & UKA & 4799 & & & & Many & \\
\hline \multirow[t]{2}{*}{ Yim JH et al. [17] } & 2012 & Retrospective & HTO & 58 & 58 & $7 / 51$ & 58.3 & OWHTO & 3.6 years \\
\hline & & & UKA & 50 & 50 & $2 / 48$ & 60.3 & Miller-Galante & 3.7 years \\
\hline S Karamitev et al. & 2014 & Retrospective & HTO & 92 & 103 & $47 / 45$ & NS & NS & NS \\
\hline [18] & & & UKA & 65 & 66 & $23 / 42$ & & & \\
\hline \multirow[t]{2}{*}{ Tuncay et al. [19] } & 2015 & Retrospective & HTO & 88 & 93 & 18/70 & 52.6 & OWHTO + Dome & 3 years \\
\hline & & & UKA & 94 & 109 & $15 / 79$ & 58.7 & Oxford & 3.5 years \\
\hline \multirow[t]{2}{*}{ Petersen et al. [20] } & 2016 & Retrospective & $\mathrm{HTO}$ & 23 & 23 & $14 / 9$ & 58.9 & OWHTO & 5 years \\
\hline & & & UKA & 25 & 25 & $9 / 16$ & 60.7 & Oxford III & 5 years \\
\hline AJ Krych et al. [21] & 2017 & Retrospective & $\begin{array}{l}\text { HTO } \\
\text { UKA }\end{array}$ & $\begin{array}{l}57 \\
183\end{array}$ & $\begin{array}{l}57 \\
183\end{array}$ & $\begin{array}{l}41 / 16 \\
82 / 101\end{array}$ & $\begin{array}{l}42.7 \\
49.2\end{array}$ & $\begin{array}{l}\text { OWHTO + CWHTO } \\
\text { Miller-Galante }\end{array}$ & $\begin{array}{l}7.2 \text { years } \\
5.8 \text { years }\end{array}$ \\
\hline
\end{tabular}

year year of publication, Type procedure type, Pts patients, Knee number of operated knee, M/F male/female, HTO high tibial osteotomy, UKA unicompartmental knee arthroplasty, CWHTO close-wedge high tibial osteotomy, OWHTO open-wedge high tibial osteotomy, PCA porous coated anatomic implant, NS not stated

yielded differing results, with $p$ values of 0.49 and 0.01 , respectively, compared to the UKA group.

\section{Pain assessment}

Five studies $[5,9,11,13,18]$ reported post-operative results for pain assessment. Patients in the UKA group tended to have better results. According to our analysis, the difference was significant $(p=0.03$; OR $=0.34 ; 95 \%$ $\mathrm{CI}=0.13,0.91)$.

\section{Deterioration}

Based on the available data, only two studies [9, 17] included information on deterioration. However, the difference for contralateral deterioration was not significant $(p=0.43 ; \mathrm{OR}=2.24 ; 95 \% \mathrm{CI}=0.30,16.72)$, nor was the difference for patellofemoral deterioration $(p=0.21$; OR $=2.01 ; 95 \% \mathrm{CI}=0.67,6.04)$.

\section{Range of motion (ROM)}

Our analysis revealed better flexion and extended ROM in the HTO group compared to the UKA group in five studies $[5,12,13,15,17]$, with $p$ values $<0.01 \quad(\mathrm{STD}=0.78$; $95 \% \mathrm{CI}=0.21,1.36)$.

\section{Free walking speed (velocity)}

Only three studies $[5,10,13]$ compared the free walking speed between HTO and UKA patients; these showed no significant difference $(p=0.66 ;$ STD $=-0.09 ; 95 \%$ 
Table 2 Summary of data recorded from studies included in meta-analysis

\begin{tabular}{|c|c|c|c|c|c|c|c|c|c|c|c|c|}
\hline Author & Type & Pts & Knee & $\begin{array}{l}\text { E/G } \\
\text { results }\end{array}$ & $\begin{array}{l}\text { Pain } \\
\text { no/mild }\end{array}$ & $\begin{array}{l}\text { Revision } \\
\text { TKA }\end{array}$ & Complication & Knee & Score & ROM & Velocity & FTA \\
\hline \multirow[t]{2}{*}{ Karpman et al. [8] } & HTO & 21 & 23 & 11 & NS & 0 & 11 & NS & NS & NS & NS & NS \\
\hline & UKA & 19 & 21 & 19 & & 2 & 3 & & & & & \\
\hline \multirow[t]{2}{*}{ Broughton et al. [9] } & HTO & 45 & 49 & 21 & 23 & 10 & 17 & Baily & $35.8 \pm 7$ & NS & NS & NS \\
\hline & UKA & 34 & 42 & 32 & 34 & 3 & 4 & & $39.6 \pm 7.3$ & & & \\
\hline Jefferson RJ et al. & HTO & 20 & 23 & NS & NS & 5 & NS & NS & NS & NS & $1.02 \pm 0.19$ & NS \\
\hline [10] & UKA & 20 & 24 & & & 17 & & & & & $0.99 \pm 0.21$ & (+) $3.2^{\circ}$ \\
\hline \multirow[t]{2}{*}{ Ivarsson et al. [5] } & HTO & 10 & 10 & 4 & 10 & NS & NS & Lysholm & $78 \pm 19$ & $121 \pm 11$ & $0.94 \pm 0.30$ & NS \\
\hline & UKA & 10 & 10 & 8 & 10 & & & & $91 \pm 11$ & $112 \pm 13$ & $0.93 \pm 0.22$ & \\
\hline \multirow[t]{2}{*}{ Weale et al. [11] } & HTO & 21 & 21 & 7 & 9 & 17 & NS & Baily & 31 & NS & NS & NS \\
\hline & UKA & 15 & 15 & 8 & 12 & 5 & & & 34 & & & \\
\hline Stukenborg et al. & HTO & 32 & 32 & 15 & NS & 10 & 9 & KSS & $76(29-100)$ & $117(85-135)$ & NS & $(-) 0.25^{\circ}$ \\
\hline [12] & UKA & 28 & 30 & 13 & & 6 & 2 & & $74(31-94)$ & $103(35-140)$ & & $(-) 5.25^{\circ}$ \\
\hline \multirow[t]{2}{*}{ Borjesson et al. [13] } & HTO & 18 & 18 & 18 & 18 & NS & NS & $\mathrm{BOA}$ & $37(36-39)$ & $123 \pm 0.5$ & $1.13 \pm 0.14$ & NS \\
\hline & UKA & 22 & 22 & 22 & 22 & & & & $37(31-39)$ & $123 \pm 0.5$ & $1.19 \pm 0.15$ & \\
\hline \multirow[t]{2}{*}{ Dettoni et al. [14] } & HTO & 54 & & 50 & NS & 0 & NS & KSS & NS & NS & NS & NS \\
\hline & UKA & 56 & & 53 & & 0 & & & NS & & & \\
\hline \multirow[t]{2}{*}{ Takeuchi et al. [15] } & HTO & 24 & 27 & 27 & NS & 0 & 2 & KSS & $89 \pm 7.6$ & $146 \pm 5.9$ & NS & $170 \pm 2.1^{\circ}$ \\
\hline & UKA & 18 & 30 & 29 & & 2 & 3 & & $79 \pm 6.8$ & $127 \pm 16$ & & $174 \pm 3.8^{\circ}$ \\
\hline \multirow[t]{2}{*}{ Dahl-W et al. [16] } & HTO & 450 & & NS & NS & 76 & NS & NS & NS & NS & NS & NS \\
\hline & UKA & 4799 & & & & 816 & & & & & & \\
\hline \multirow[t]{2}{*}{ Yim JH et al. [17] } & HTO & 58 & 58 & NS & NS & NS & 3 & Lysholm & $89.6 \pm 8.7$ & $138.8 \pm 4.7$ & NS & (+) $1.8 \pm 1.7^{\circ}$ \\
\hline & UKA & 50 & 50 & & & & 3 & & $90.3 \pm 7.7$ & $130.0 \pm 8.8$ & & $(-) 1.9 \pm 2.2^{\circ}$ \\
\hline S Karamitev et al. & HTO & 92 & 96 & 83 & 78 & NS & NS & KSS & NS & NS & NS & NS \\
\hline [18] & UKA & 65 & 66 & 65 & 56 & & & & & & & \\
\hline \multirow[t]{2}{*}{ Tuncay et al. [19] } & HTO & 88 & 93 & NS & NS & 0 & 8 & HSS & 83.73 & NS & NS & NS \\
\hline & UKA & 94 & 109 & & & 3 & 3 & & 90 & & & \\
\hline \multirow[t]{2}{*}{ Petersen et al. [20] } & HTO & 23 & 23 & 17 & NS & 1 & 2 & HSS & NS & NS & NS & NS \\
\hline & UKA & 25 & 25 & 21 & & 1 & 1 & & & & & \\
\hline \multirow[t]{2}{*}{ AJ Krych et al. [21] } & HTO & 57 & 57 & NS & NS & 13 & NS & Lysholm & $80.2 \pm 11.8$ & NS & NS & $(+) 1.3 \pm 2.4^{\circ}$ \\
\hline & UKA & 183 & 183 & & & 11 & & & $90.0 \pm 11.0$ & & & NS \\
\hline
\end{tabular}

Type procedure type, Pts patients, Knee number of operated knee, E/G excellent, good result, Pain pain assessment, ROM range of motion, Velocity free walking speed, FTA femoro-tibial angle, Baily Baily knee score, Lysholm Lysholm knee score, KSS Knee Society score, BOA British Orthopaedic Association score, HSS Hospital for Special Surgery score, (+) valgus, (-) varus, NS not stated

$\mathrm{CI}=-0.48,0.30$ ).

\section{Knee score}

Seven studies $[5,12,13,15,17,19,21]$ used various scoring systems to compare knee scores between the two procedures. Although no statistically significant difference was found ( $p=0.11 ; \mathrm{STD}=-0.21 ; 95 \% \mathrm{CI}=-0.47,0.05)$, the UKA group exhibited better functional results. Our study also analyzed the Lysholm knee score $[5,17,21]$ and Knee society score (KSS) [12, 15], which showed no significant differences ( $p=0.08$ and 0.59 , respectively).

\section{Complication}

Generally, more complication were noted after a valgus HTO with significant difference found between the two groups $(p<0.001$; $\mathrm{OR}=3.08 ; 95 \% \mathrm{CI}=1.76,5.39)$, reflecting results from seven studies $[8,9,12,15,17,19,20]$ with 559 patients.

\section{Revision}

Eleven studies [8-12, 14-16, 19-21] with 6241 patients reported revisions. The pooled data showed no significant difference between HTO and UKA in terms of revision rate $(p=0.68 ; \mathrm{OR}=1.18 ; 95 \% \mathrm{CI}=$ 0.54, 2.58; Fig. 5). 
Table 3 Result of the meta-analysis

\begin{tabular}{|c|c|c|c|c|c|c|c|c|}
\hline \multirow[t]{2}{*}{ Outcome } & \multirow[t]{2}{*}{ Studies } & \multicolumn{2}{|c|}{ Sample size } & \multirow{2}{*}{$\begin{array}{l}\text { Effect estimate } \\
\text { Odds ratio }(95 \% \mathrm{Cl})\end{array}$} & \multirow[t]{2}{*}{ P } & \multirow{2}{*}{$\begin{array}{l}\text { Effect estimate } \\
\text { STD }(95 \% \text { Cl) }\end{array}$} & \multicolumn{2}{|c|}{ Heterogeneity } \\
\hline & & HTO & $\overline{U K A}$ & & & & $P(\%)$ & $\mathrm{Chi}^{2}(P)$ \\
\hline Pain assesment (no/mild) & 5 & 194 & 155 & $0.34[0.13,0.91]$ & 0.03 & & 61 & 0.08 \\
\hline Excellent/good (E/G) result & 10 & 353 & 317 & $0.37[0.24,0.58]$ & $<0.00001$ & & 39 & 0.11 \\
\hline Excellent/good result (medial OA/varus) & 5 & 110 & 117 & $0.75[0.37,1.52]$ & 0.43 & & 19 & 0.29 \\
\hline Subgroup: E/G CWHTO-UKA & 6 & 153 & 140 & $0.36[0.21,0.61]$ & 0.01 & & 56 & 0.06 \\
\hline Subgroup: E/G OWHTO-UKA & 3 & 104 & 111 & $0.70[0.26,1.91]$ & 0.49 & & 0 & 0.66 \\
\hline Knee score & 7 & 262 & 317 & & 0.11 & $-0.21[-0.47,0.05]$ & 51 & 0.05 \\
\hline Lysholm knee score & 3 & 92 & 126 & & 0.08 & $-0.53[-1.12,0.06]$ & 71 & 0.03 \\
\hline Knee Society Score (KSS) & 2 & 59 & 60 & & 0.59 & $0.10[-0.26,0.46]$ & 0 & 0.88 \\
\hline Deterioration of contralateral & 2 & 107 & 92 & $2.24[0.30,16.72]$ & 0.43 & & 74 & 0.05 \\
\hline Deterioration of patellofemoral & 2 & 107 & 92 & $2.01[0.67,6.04]$ & 0.21 & & 0 & 0.57 \\
\hline ROM & 5 & 145 & 142 & & 0.008 & $0.78[0.21,1.36]$ & 80 & 0.0005 \\
\hline Velocity & 3 & 51 & 51 & & 0.66 & $-0.09[-0.48,0.30]$ & 0 & 0.44 \\
\hline Complication & 7 & 305 & 307 & $3.08[1.76,5.39]$ & $<0.0001$ & & 7 & 0.37 \\
\hline Revision rate & 11 & 880 & 5361 & $1.18[0.54,2.58]$ & 0.68 & & 74 & $<0.0001$ \\
\hline
\end{tabular}

HTO high tibial osteotomy, UKA unicompartmental knee arthroplasty, $P \mathrm{p}$ value, E/G excellent, good result, OA osteoarthritis, Varus varus deformity, STD Std mean difference, $\mathrm{Cl}$ confidence interval

\section{Discussion}

OA affects any or all three compartments of the knee. However, one third of patients are afflicted in only one of these compartments, many of them having a medial compartment disorder [22].

The purpose of surgery for unicompartment $\mathrm{OA}$ is to reduce pain, restore function, and improve the patient's quality of life. The most important finding of this study was that both HTO and UKA are satisfactory operative treatment options for symptomatic medial knee OA.

Patient selection is generally stricter for individuals undergoing HTO than for those receiving UKA. However, medial knee arthritis patients selected for HTO experience many benefits. Ideal indications for HTO include (1) young and active patients (age <65 years) [23, 24], (2) normal-range body mass index (BMI) [25], (3) mild articular destruction (no more than grade 2 Ahlbäck classification), (4) no patellofemoral arthrosis [26], and (5) good ROM and a stable joint [27].

Age, BMI, and pre-operative state OA are key factors that optimize clinical outcomes and survival in patients undergoing HTO. Previous studies have reported that a pre-operative BMI higher than 27.5 is a significant risk factor for early failure [25], and patients with BMI over 30 exhibit significantly lower KSS and WOMAC scores 5 years after HTO [28]. Moreover, HTO is not advisable

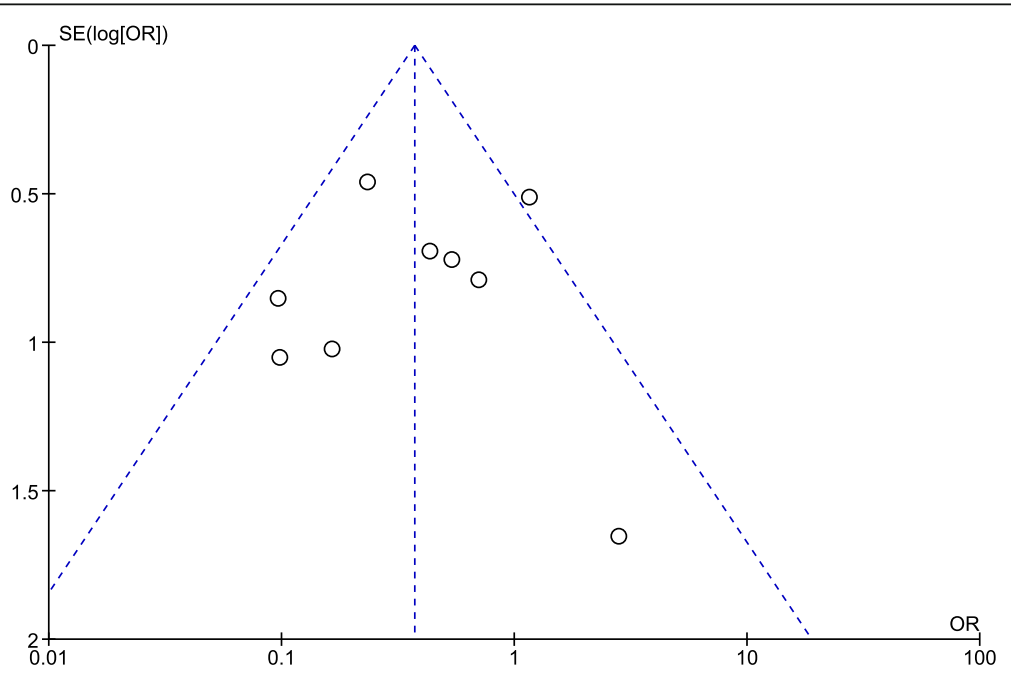

Fig. 3 Funnel plot to assess small study exclusion/publication bias 


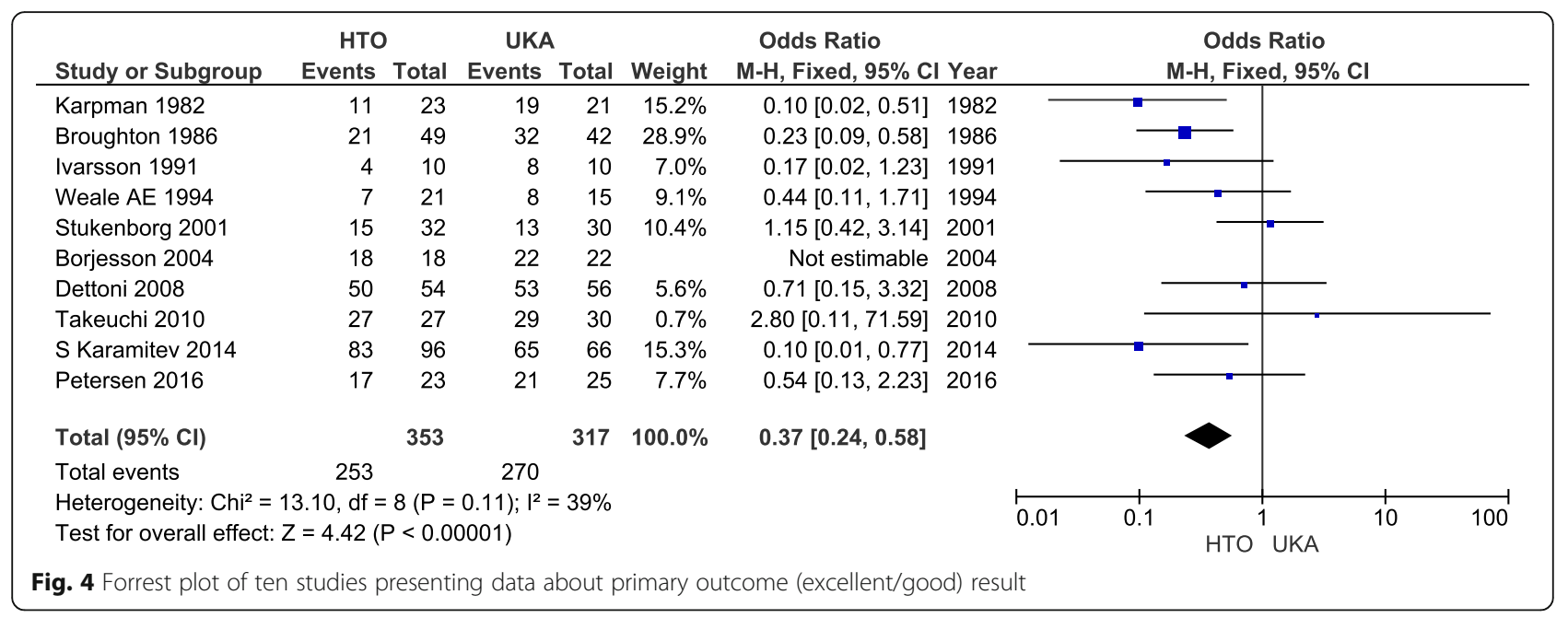

for patients older than 65 years due to the $7.6 \%$ increased risk per year of age and the 1.5-fold relative risk of failure compared to younger patients [29].

HTO and UKA share similar indications that include the following: age 55-65 years, moderately active, nonobese, presenting with mild varus malalignment and moderate unicompartmental arthrosis, no joint instability, and good ROM [30].

Indications for UKA are broadening after reports of promising mid- and long-term results, which include isolated medial or lateral compartment $\mathrm{OA}$, osteonecrosis of the knee, age over 60 years, weight under $82 \mathrm{~kg}$, and an ideal ROM of 90 with fewer than $5^{\circ}$ flexion contractures. Contraindications include high activity, age under 60 years, and inflammatory arthritis [11, 30, 31].

Our analysis demonstrated a significant difference in outcomes between UKA and HTO patients, with the former showing better functional results (excellent/good results), and the latter better ROM. This discrepancy was correlated with knee score and ROM, indicating the possibility of additional impacts on the functional results.

Earlier publications reported a valgus deformity treated with either procedure. In our opinion, the clinical results for patients with a surgically treated valgus deformity, by either arthroplasty or osteotomy, cannot be compared to results for patients with a varus deformity. Major differences between medial and lateral UKA, as well as between varus and valgus osteotomy have been noted [4, 9, 32-34]. Therefore, our analysis showing excellent/good functional results focused only on studies with a strict inclusion criterion of medial knee OA with a varus deformity; analysis of such cases showed no significant difference between the two procedures. The subgroup analysis yielded similar findings and revealed favorable results in the UKA group relative to closed-wedge HTO (CWHTO) patients; however, these results were not noted when comparing open-wedge HTO (OWHTO) and UKA. CWHTO was

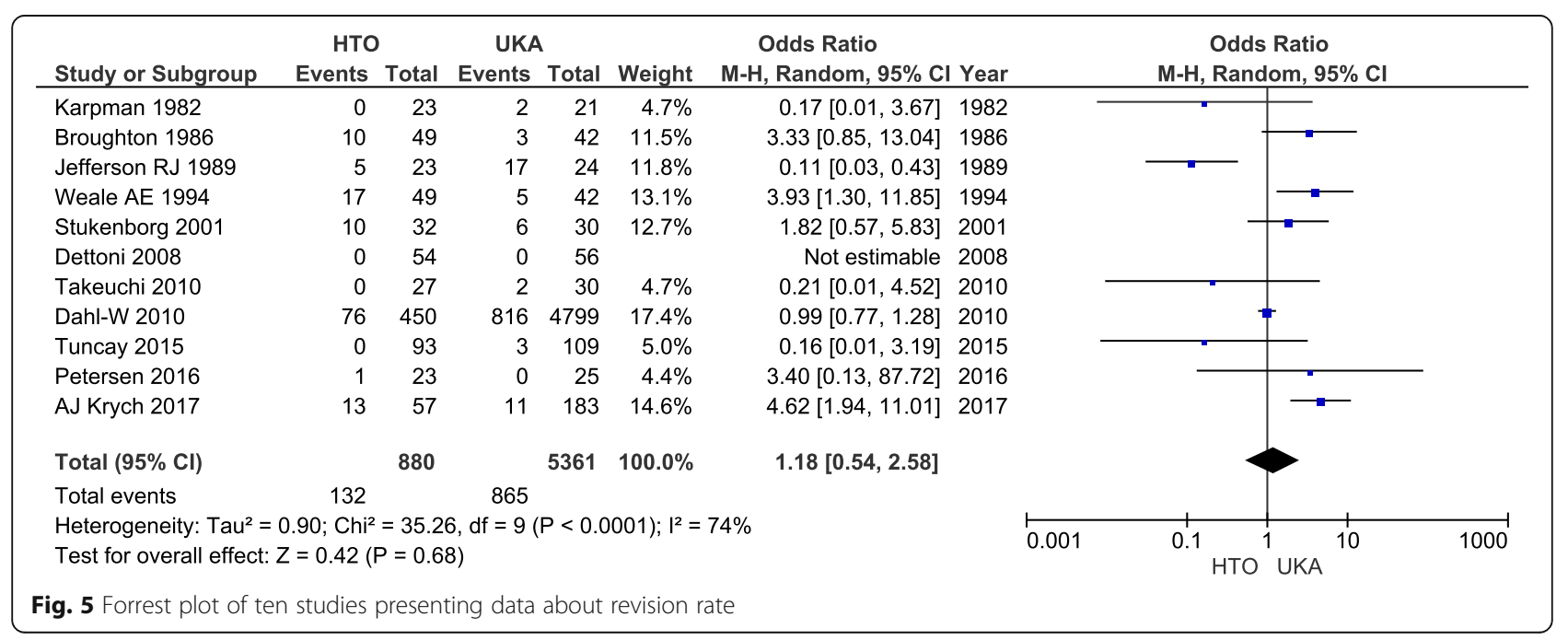


the main treatment method for HTO in the past, but OWHTO was recently reported to yield good or excellent results, owing to improvements in surgical techniques and implant stability [35, 36]. However, recent meta-analyses comparing CWHTO and OWHTO did not report superiority of OWHTO over CWHTO [37, 38].

A greater change in ROM was noted in the UKA group relative to the HTO group due to a lower preoperative score [5]. Takeuchi et al. [15] reported that OWHTO is a more appropriate treatment method for active patients who require good ROM of the knee. The unsatisfactory results of the HTO group were mostly due to an insufficient deformity correction. Previous studies reported that optimal results can be achieved if the mechanical alignment is adjusted to $7^{\circ}$ [39]. Nevertheless, the ultimate post-operative valgus position is technically challenging to achieve.

Free walking speed (velocity) has been proven both a reliable and a valid indicator to evaluate treatment outcomes in knee OA patients [40, 41]. Our meta-analysis found no significant difference between the two procedures in terms of velocity $(p=0.66)$, although $\mathrm{Fu}$ et al. [42] reported otherwise $(p=0.05)$. However, given that both studies used the same literature to arrive at this outcome, differing results were not expected. It is also important to note that Jefferson et al. [10] assessed the velocity outcome of three operative methods (HTO, UKA, and TKA), with post-operative results reported as $1.02 \pm 0.19,0.99 \pm 0.21$, and $0.81 \pm 0.19 \mathrm{~m} / \mathrm{s}$, respectively. However, Fu et al. [42] included the TKA results (0.81 \pm 0.19 ) in their analysis of the HTO group, which may suggest an inaccuracy. Therefore, our results are more accurate and reliable.

Our analysis revealed that free walking speed was improved after both HTO and UKA but with an equivalent rise in the UKA group. Borjesson et al. [13] stated that, compared to HTO patients, UKA patients had a greater increase in free walking speed, with results 5 years after surgery that were highly similar to the walking speed of healthy people of the same age group [43]. Moreover, both procedures resulted in an almost normal gait pattern.

Ivarsson et al. [5] showed that UKA patients have better muscle strength than do HTO patients 6 months postoperatively, but the 12-month post-operative results were similar. One explanation for this finding is that rehabilitation of UKA patients normally begins earlier, whereas HTO patients usually undergo an immobilization period. Moreover, HTO patients may require a longer time to adapt due to greater changes in post-surgical leg alignment.

Regarding the progression of knee OA, our analysis showed that the OR of the risk of contralateral and patellofemoral deterioration did not differ between groups, although the HTO group tended to exhibit this problem. One logical explanation is that this phenomenon is due to the overcorrection to unleash the medial compartment during the procedure, thus suppressing the lateral compartment and leading to deterioration. Overcorrection of more than $6^{\circ}$ was associated with progressive degeneration of the lateral compartment [44]. In addition, OWHTO above the tibial tubercle can have adverse effects on patellofemoral articulation [2, 45, 46]. Yim et al. [17] compared OWHTO and UKA patients and reported that two cases of UKA showed patellofemoral joint OA compared to three cases of OWHTO.

Compared to UKA, the chance of post-operative complications is greater after an osteotomy [39]. Our analysis revealed a significant difference in such complications between HTO and UKA patients, supporting previous studies and a meta-analysis by Spahn et al. [47]. Among all included studies in this present study, five trials applied OWHTO, seven trials used CWHTO, and one study used hemicallotasis. OWHTO is considered safe and easy [21, 48, 49] based on the assumption that CWHTO may be associated with a higher incidence of complications, especially peroneal nerve paralysis. Despite improved surgical techniques and implant design, previous studies have reported complications after UKA, such as loosening of the tibial or femoral component or osteoarthritic changes in the development of the lateral compartment due to antero-posterior instability of the knee, which leads to rapid wearing of the polyethylene insert $[11,21]$. In the HTO group, most complications were associated with an intra-articular fracture, nonunion, infection, and peroneal nerve palsy.

TKA is defined as a clear end-point after both HTO and UKA. Medial UKA patients tend to require revision sooner [21], with a mean of 8.2 years compared to a mean of 9.7 years for valgus HTO patients [47]. Barrett and Scott [50] reported 29 unsuccessful UKA revisions to TKA and observed that the mechanism of failure was loosening in 55\% of cases and degeneration advancement of the remaining compartments in $31 \%$ of patients. Technical errors during the primary UKA and poor selection of patients contributed to $66 \%$ of failures.

Cross et al. [51] examined the operative time and found that revision to TKA in HTO patients required more time compared to that for UKA patients, which could be because the HTO procedure is complicated by difficulties in obtaining an acceptable exposure, removing retained hardware, achieving correct tibial component positioning, scarring, and additional challenges with ligamentous balancing that have been reported to result from a prior HTO. The major technical difficulty in the revision UKA group was handling the bony defects on both the tibial and femoral sides. Significantly thicker polyethylene inlays were required during the 
revision of UKA to TKA compared to primary TKA [52], and the UKA group required substantially more osseous reconstruction (77\%) compared to the HTO group (20\%) [30].

Consistent with the previous meta-analysis [42], the present study also failed to identify any significant difference in the revision rate between the two procedures. Although both groups exhibited higher revision rates over time with deteriorated clinical outcomes, the risk of revision of primary UKA declined with age. The 10-year revision rate was nearly $24 \%$ in patients aged less than 55 years, threefold higher than that in those aged 55 years and older [16].

Robertsson et al. [53] reported that hospitals that perform 23 or more UKAs per year have a 1.6-fold lower revision rate compared to those who perform fewer than 23. Therefore, routine patient selection and good surgical skills are believed to influence the results of the UKA procedure; this principle may also apply to HTO.

Several limitations of this study should be noted. First, a controlled randomized trial is challenging due to ethical concerns. The present meta-analysis included only three randomized controlled trials of the 15 studies and the patients enrolled for HTO tended to be younger than those enrolled for UKA. Although most studies reported good numbers, the use of diverse analyzing systems and methods can lead to difficulties comparing and assembling the outcomes, as well as inability to evaluate essential items such as radiographic changes due to inadequate data. Moreover, the current analysis showed that UKA and HTO are distinct in terms of their techniques and indications for patients with medial unicompartmental OA. Finally, the small patient population made it difficult to compare the two procedures and arrive at a conclusion regarding the clinical outcomes.

\section{Conclusions}

In conclusion, valgus $\mathrm{HTO}$ is a technically challenging procedure but provides younger OA patients with good physical activity. On the other hand, UKA is more suitable for older patients, as it provides a greater quality of life with a shorter rehabilitation time required before full weight bearing, fewer perioperative complications, and faster functional recovery compared to HTO.

Accurate identification of indications, including age, level of activity, grade of OA and ROM of the knee, and careful patient selection are essential for all OA patients. Nevertheless, with advancements in surgical techniques, implant design and patient selection, UKA has become a more reliable and effective procedure.

Finally, although UKA patients tended to have improved overall long-term outcomes, both treatment options offered pleasing results, and no significant evidence supports one method over the other. Additional well- designed and large-scale clinical trials and systematic reviews are necessary to confirm the findings presented here.

\section{Abbreviations}

BMI: Body mass index; Cl: Confidence interval; CWHTO: Closing-wedge high tibial osteotomy; HTO: High tibial osteotomy; KSS: Knee society score; OA: Osteoarthritis; OR: Odds ratio; OWHTO: Opening-wedge high tibial osteotomy; ROM: Range of motion; STD: Standardized mean difference TKA: Total knee arthroplasty; UKA: Unicompartmental knee arthroplasty

\section{Acknowledgements}

This study was supported by the Department of Orthopedic Surgery of the Second Affiliated Hospital of Zhejiang University for their assistance in obtaining the texts, which formed the basis of this meta-analysis.

Funding

No funding source was involved in the conduction of this study.

\section{Availability of data and material}

The dataset(s) supporting the conclusions of this article are included within the article.

Authors' contributions

MBS and LDW are both contribute for the concepts, design, literature search, data acquisition, data and statistical analysis, manuscript preparation. LDW also acts as a consultant and guarantor of this study. Both authors read and approved the final manuscript.

Competing interests

The authors declare that they have no competing interests.

Consent for publication

Not applicable

Ethics approval and consent to participate

Not applicable

\section{Publisher's Note}

Springer Nature remains neutral with regard to jurisdictional claims in published maps and institutional affiliations.

Received: 27 December 2016 Accepted: 17 March 2017

Published online: 28 March 2017

References

1. Jackson JP. Osteotomy for osteoarthritis of the knee. J Bone Joint Surg (Br). 1958:40:826.

2. Gaasbeek R, Welsing R, Marink M, Verdonschot N, van Kampen A. The influence of open and closed high tibial osteotomy on dynamic patellar tracking: a biomechanical study. Knee Surg Sports Traumatol Arthrosc. 2007;5:978-84.

3. Hohmann E, Bryant A. Closing or opening wedge high tibial osteotomy: watch out for the slope. Orth Tech Orthop. 2007;17:38-45.

4. Insall J, Aglietti P. A 5- to 7-year follow-up of unicondylar arthroplasty. J Bone Joint Surg Am. 1980;62:1329.

5. Ivarsson I, Gillquist J. Rehabilitation after high tibial osteotomy and unicompartmental arthroplasty: a comparative study. Clin Orthop Relat Res. 1991;266:139.

6. Higgins JPT, Thompson SG, Deeks JJ, Altman DG. Measuring inconsistency in meta analyses. BMJ. 2003:327:557.

7. Mantel N, Haenszel W. Statistical aspects of the analysis of data from retrospective studies of disease. J Natl Cancer Inst. 1959;22:719.

8. Karpman RR, Volz RG. Osteotomy versus unicompartmental prosthetic replacement in the treatment of unicompartmental arthritis of the knee. Orthopedics. 1982;5(8):989.

9. Broughton NS, Newman JH, Baily RA. Unicompartmental replacement and high tibial osteotomy for osteoarthritis of the knee. A comparative study after 5-10 years' follow-up. J Bone Joint Surg (Br). 1986;68(3):447. 
10. Jefferson RJ, Whittle MW. Biomechanical assessment of unicompartmental knee arthroplasty, total condylar arthroplasty and tibial osteotomy. Clin Biomech. 1989:4(4):232.

11. Weale AE, Newman JH. Unicompartmental arthroplasty and high tibial osteotomy for osteoarthrosis of the knee. Clin Orthop Relat Res. 1994;302:134.

12. Stukenbor C, Wirth CJ, Lazovic D, et al. High tibial osteotomy versus unicompartmental joint replacement in unicompartmental knee joint osteoarthritis: 710-year follow-up prospective randomised study. Knee. 2001;8:187.

13. Borjesson M, Weidenhielm L, Mattsson E, et al. Gait and clinical measurements in patients with knee osteoarthritis after surgery: a prospective 5-year follow-up study. Knee. 2005;12:121. doi:10.1016/j.knee. 2004.04.002.

14. Dettoni F, Maistrelli GL, Rossi $\mathrm{P}$, et al. UKA versus HTO: clinical results at short term follow up. 75th AAOS Annual Meeting. 2008. San Francisco, CA; 2008.

15. Takeuchi $R$, Umemoto $Y$, Aratake $M$, et al. A mid term comparison of open wedge high tibial osteotomy vs unicompartmental knee arthroplasty for medial compartment osteoarthritis of the knee. J Orthop Surg Res. 2010;5:65.

16. Dahl AW, Robertsson O, Lidgren L. Surgery for knee osteoarthritis in younger patients. A Swedish register study. Acta Orthop. 2010;81(2):161. doi:10.3109/17453670903413186.

17. Yim JH, Song EK, Seo HY, et al. Comparison of high tibial osteotomy and unicompartmental knee arthroplasty at a minimum follow-up of 3 years. J Arthroplasty. 2013;28(2):243.

18. Karamitev S, Stavrev VP, Chifligarov AG. Comparative analysis of the result obtained after unicondylar knee arthroplasty and high tibial osteotomy in isolated gonarthrosis. Folia Med. 2014;56(1):11-9. doi:10. 2478/folmed-2014-0002.

19. Tuncay I, Bilsel K, Elmadag M, et al. Evaluation of mobile bearing unicompartmental knee arthroplasty, opening wedge, and dome-type high tibial osteotomies for knee arthritis. Acta Orthop Traumatol Turc. 2015;49(3): 280-7. doi:10.3944/AOTT.2015.14.0320

20. Petersen W, Metzlaff S. Open wedge high tibial osteotomy (HTO) versus mobile bearing unicondylar medial joint replacement: five years results. Arch Orthop Trauma Surg. 2016;136:983-9. doi:10.1007/s00402-016-2465-1.

21. Krych AJ, Reardon P, Sousa P, Pagnano M, et al. Unicompartmental knee arthroplasty provides higher activity and durability than valgus-producing proximal tibial osteotomy at 5 to 7 years. J Bone Joint Surg Am. 2017;99: 113-22. doi:10.2106/JBJS.15.01031

22. Ledingham J, Regan M, Jones A, et al. Radiographic patterns and associations of osteoarthritis of the knee in patients referred to hospital. Ann Rheum Dis. 1993;52:520-6.

23. Flecher $X$, Parratte $S$, Aubaniac JM, et al. A 12-28-year follow-up study of closing wedge high tibial osteotomy. Clin Orthop Relat Res. 2006;452:91.

24. Gstottner M, Pedross F, Liebensteiner $M$, et al. Long-term outcome after high tibial osteotomy. Arch Orthop Trauma Surg. 2008;128(1):111.

25. Akizuki S, Shibakawa A, Takizawa T, Yamazaki I, Horiuchi H. The long-term outcome of high tibial osteotomy: a ten- to 20-year follow-up. J Bone Joint Surg (Br). 2008;90(5):592-6.

26. Rudan JF, Simurda MA. High tibial osteotomy. A prospective clinical and roentgenographic review. Clin Orthop Relat Res. 1990;255:251.

27. Amendola A, Bonasia DE. Results of high tibial osteotomy: review of the literature. Int Orthop. 2010;34(2):155.

28. Howells NR, Salmon L, Waller A, Scanelli J, Pinczewski LA. The outcome at ten years of lateral closing-wedge high tibial osteotomy: determinants of survival and functional outcome. Bone Joint J Br. 2014;96(11):1491-7.

29. Trieb K, Grohs J, Hanslik-schnabel B, et al. Age predicts outcome of high-tibial osteotomy. Knee Surg Sports Traumatol Arthrosc. 2006;14(2):149.

30. Dettoni F, Bonasia DE, Castoldi F, et al. High tibial osteotomy versus unicompartmental knee arthroplasty for medial compartment arthrosis of the knee: a review of the literature. Owa Orthop J. 2010;30:131.

31. Borus T, Thornhill T. Unicompartmental knee arthroplasty. J Am Acad Orthop Surg. 2008;16(1):9.

32. Laskin RS. Unicompartmental tibiofemoral resurfacing arthroplasty. J Bone Joint Surg. 1978;60-A:182-5.

33. Tjörnstrand BA, Egund N, Hagstestedt B. High tibial osteotomy: a seven-year clinical and radiographic follow-up. Clin Orthop. 1981;160:124-36.

34. Shoji H, Insall JN. High tibial osteotomy for osteoarthritis of the knee with valgus deformity. J Bone Joint Surg. 1973;55-A:963-73.

35. Kolb W, Guhlmann H, Windisch C, et al. Opening-wedge high tibial osteotomywith a locked low-profile plate. J Bone Joint Surg Am. 2009;91:2581.
36. Staubli AE, De Simoni C, Babst R, et al. TomoFix: a new LCP concept for open wedge osteotomy of the medial proximal tibia-early results in 92 cases. Injury. 2003;34 Suppl 2:B55.

37. Smith TO, Sexton D, Mitchell P, Hing CB. Opening- or closing-wedged high tibial osteotomy: a meta-analysis of clinical and radiological outcomes. Knee. 2011;18:361-8. doi:10.1016/j.knee.2010.10.001.

38. Hao Sun, Lin Zhou, Fengsheng Li, Jun Duan, Comparison between closing-wedge and opening-wedge high tibial osteotomy in patients with medial knee osteoarthritis: a systemic review and meta-analysis. J Knee Surg. DOl: http://dx.doi.org/10.1055/s-0036-1584189. ISSN 1538-8506.

39. Kozinn SC, Scott RD. Unicondylar knee arthroplasty. J Bone Joint Surg. 1989;71-A:145

40. Marks R. Reliability and validity of self-paced walking time measures for knee osteoarthrosis. Arthritis Care Res. 1994;7(1):50-3.

41. Fransen M, Crosbie J, Edmonds J. Reliability of gait measurements in people with osteoarthritis of the knee. Phys Ther. 1997;77(9):944-53.

42. Fu D, Li G, Chen K, Zhao Y, Hua Y, Cai Z. Comparison of high tibial osteotomy and unicompartmental knee arthroplasty in the treatment of unicompartmental osteoarthritis: a meta-analysis. J Arthroplasty. 2013;28(5):759-65.

43. Bohannan RW, Williams Andrews A, Thomas MW. Walking speed: reference values and correlates for older adults. J Orthop Sports Phys Ther. 1996;24(2):86-9

44. Hernigou P, Medevielle D, Debeyre J, Goutallier D. Proximal tibial osteotomy for osteoarthritis with varus deformity. A ten to thirteen-year follow-up study. J Bone Joint Surg Am. 1987;69:332-54.

45. Brouwer RW, Bierma-Zeinstra SM, van Koeveringe AJ, et al. Patellar height and the inclination of the tibial plateau after high tibial osteotomy. The open versus the closedwedge technique. J Bone Joint Surg (Br). 2005;87:1227.

46. Stoffel K, Willers C, Korshid O, et al. Patellofemoral contact pressure following high tibial osteotomy: a cadaveric study. Knee Surg Sports Traumatol Arthrosc. 2007;15:1094

47. Spahn G, Hofmann GO, Engelhardt LV, et al. The impact of a high tibial valgus osteotomy and unicondylar medial arthroplasty on the treatment for knee osteoarthritis: a meta-analysis. Knee Surg Sports Traumatol Arthrosc. 2013;21:96-112. doi:10.1007/s00167-011-1751-2.

48. Coventry MB, Ilstrup DM, Wallrichs SL. Proximal tibial osteotomy, a critical long term study of eighty-seven cases. J Bone Joint Surg Am. 1993;75:196-201.

49. Brinkman JM, Lobenhoffer $P$, Agneskirchner JD, Staubli AE, Wymenga AB, van Heerwaarden RJ. Osteotomies around the knee: patient selection, stability of fixation and bone healing in high tibial osteotomies. J Bone Joint Surg (Br). 2008;90:1548-57.

50. Barrett WP, Scott RD. Revision of failed unicondylar unicompartmental knee arthroplasty. J Bone Joint Surg Am. 1987;69:1328.

51. Cross MB, Paul YY, Moric M, et al. Revising an HTO or UKA to TKA: is it more like a primary TKA or a revision TKA? J Arthroplasty. 2014;29(2):229-31.

52. Gill T, Schemitsch EH, Brick GW, et al. Revision total knee arthroplasty after failed unicompartmental knee arthroplasty or high tibial osteotomy. Clin Orthop Relat Res. 1995;321:10.

53. Robertsson O, Knutson K, Lewold S, Lidgren L. The routine of surgical management reduces failure after unicompartmental knee arthroplasty. J Bone Joint Surg (Br). 2001;83(1):45-9.

\section{Submit your next manuscript to BioMed Central and we will help you at every step:}

- We accept pre-submission inquiries

- Our selector tool helps you to find the most relevant journal

- We provide round the clock customer support

- Convenient online submission

- Thorough peer review

- Inclusion in PubMed and all major indexing services

- Maximum visibility for your research

Submit your manuscript at www.biomedcentral.com/submit 